\title{
A New Model for Evaluating the Future Options of Integrating Ground Source Heat Pumps in Building Construction
}

\author{
Claudio Martani, Ying Jin, Kenichi Soga and Stefan Scholtes \\ CSIC, University of Cambridge, UK
}

\begin{abstract}
Decision-making for effective infrastructure integration is challenging because the performances of long-lasting objects often depends on conditions which are either outside the control of the designer or difficult to foresee at the design stage. In this paper we examine a new approach to estimating the range of cost-effective solutions for integrating the construction/retrofit of two or more different types of infrastructure. Infrastructure integration has many perceived benefits, but also faces serious new challenges and doubts from practitioners, particularly in sectors with complex construction process, long asset lives, uncertain cost parameters, and slow and unwieldy decision-making, such as is common with civil engineering works. We test all main options in integrating a ground source heat pump (GSHP) system with the construction and retrofit of an archetypal, office building. A new simulation model is developed and parameterized using actual data in the UK. We incorporate unavoidable uncertainties and randomness in how the decisions are triggered, and test the effectiveness of proactive measures to embed future options. The model highlights how sensitive the range of cost-effective solutions is to the setting of renewable energy incentives, discount rates, technical performance and life-cycle asset management of interdependent infrastructure. This points to a clear need for establishing appropriate regulatory standards. We expect this model to find increasing applications in the planning and designing of integrated complexes of buildings, transport facilities, renewable energy supply, water supply and waste management in dense urban areas, which are an increasingly key part of sustainable urban development.
\end{abstract}

Keywords: Flexible Design, Options Imbedded Design, Infrastructure Integration, Resilience, GSHP, LCC, Monte Carlo Simulations

\section{INTRODUCTION}

In this paper we develop and test a new, generic modeling approach for a realistic assessment of the costs and benefits of integrating the construction/retrofit of two or more different types of infrastructure. Infrastructure integration have attracted increasing attention across different sectors ranging from building works, energy, transport, water, waste to ICT, for potentially large benefits in cutting costs, reducing key resource consumption, improving service quality and achieving wider societal benefits. However, integrative planning and design also face serious challenges that arise from hitherto unfamiliar conflicts in scheduling investment and coordinating construction and operations. Furthermore, infrastructure investors and designers (either public or private) often have reasons to doubt whether infrastructure integration would bring real, net benefits, particularly in sectors where construction process is already highly complex, expected service life long, costs uncertain and the decision-making process often slow and unwieldy, such as is common with civil engineering works.

We develop the new model with the above challenges in mind. In particular we aim to make a new extension to 
the future options approach such as put forward by Ellingham and Fawcett (2006) ${ }^{1}$ and de Neufville and Scholtes $(2011)^{2}$. The model aims to assess in a realistic and robust way the costs and benefits of embedding construction and retrofit options to enable the integration of different types of infrastructure. The model is designed in particular to address the challenges arising from construction process conflicts, long expected life cycles and significant uncertainties in costs and decision-making processes. We expect this model to find increasing use in the planning and designing of integrated complexes of buildings, transport facilities, renewable energy supply, water supply and waste management, such as necessary in dense urban areas and large building complexes.

\section{INTRODUCTION TO MODEL APPLICATION}

A new simulation model is developed and parameterized using actual data in the UK to test realistic combinations of energy prices, engineering and building system performance and decision-making processes. We incorporate unavoidable uncertainties and randomness in how the decisions are triggered, and test the effectiveness of proactive measures to embed future options in engineering and building design. The model provides a systematic assessment of the key dimensions to decision making. It highlights how sensitive the range of cost-effective solutions is to the setting of renewable energy incentives, cash flow discount rates, the technical performance of GSHP systems and a proper life-cycle asset management of interdependent infrastructure. The conclusions point to a clear need for establishing smart standards in engineering, planning and governance for exploiting the technical potential of the GSHP systems and achieving robust financial and energy savings.

GSHP is among the most important options under consideration in decarbonising building energy use, especially in

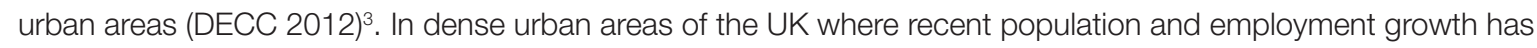
been significant, incorporating GSHP has proved to be a major challenge. After some unsuccessful pilot projects to install and operate GSHP systems in high profile office buildings, there is a widely held belief that GSHP systems are not cost effective, particularly given the uncertainties in future energy prices. Few businesses have the intention currently to implement GSHP, except in a very small number of flagship green building projects. Nevertheless, a large number of businesses and developers are concerned with the likely rise in future energy prices as well as CO2 emissions, and wish to incorporate more sustainable energy solutions so long as there is a robust financial justification of their investments.

Given the high levels of uncertainties over energy prices, GSHP system performance and financial constraints, decision making for long-lasting investments such as the GSHP systems is challenging. Indeed, the decision to install and operate GSHP systems is subject to uncertainties across many factors. Furthermore, the long-term nature of the investment makes it necessary to consider how the designs and installations can adapt to different future circumstances.

The model application aims therefore to establish a generic approach for assessing the integration, taking appropriate account of the exogenous trends and the partial and fragmented nature of their projections. In other words, it aims to define how much flexibility there should be to bring justifiable financial returns on investment in light of the uncertainty of the future circumstances and the cost of creating and exercising the options.

\section{CORE PROBLEM AND TEST SCENARIOS}

The core problem of integrating a GSHP system with an office building can be translated into a modeling process where the decision-maker faces uncertainties of a number of exogenous conditions. Scenarios that represent different future option designs and levels of flexibility (Figure 1) are then tested to evaluate the profile of financial costs and benefits. The three principal test scenarios are:

Scenario 1 - 'All Initial': All the GSHP system is included when constructing the office building. This consists of a total investment of the GSHP system at $£ 1,760,000$ ( $£ 1,200,000$ for 203 boreholes and bars, and $£ 560,000$ for the heat pump equipment). 127 bars costing $£ 750,000$ are placed in the gardens and 76 bars costing $£ 450,000$ are placed under the foundation of the building. Because the underground works take place prior to the foundation of the

1 Ellingham I., Fawcett W. (2006) New generation whole life costing, Taylor and Francis, London

2 De Neufville, R., Scholtes, S. (2011), Flexibility in Engineering Design. MIT press, Cambridge (MA)

3 DECC (2012), Fossil fuel Price projection, Department of Energy and Climate Change London 
office building, it incurs the lowest unit cost to make the boreholes and install the bars. It is also possible to put in a maximum number of boreholes on the site at a low cost. The GSHP will supply cooling, heating and hot water loads in the building.

Scenario 2 - 'No Initial': No GSHP is included when constructing the office building. The grounds that are not covered by the building's foundation can potentially allow 77 bars to be put in at a later date, at a cost of $£ 454,800$ (in today's prices, ditto below); if this option is taken up at a later date, a smaller heat pump $(£ 215,000)$ will be required. This gives a total cost of $£ 669,800$ and will supply $38 \%$ of the total building energy load. Any loads that are not covered by the GSHP system are supplied by the conventional systems, i.e. the most efficient boiler and the most efficient electrical air conditioning system as currently available.

Scenario 3 - 'Flex Initial': A smaller initial investment is made to put in 122 bars at $£ 721,200$ under the building's foundation. If at a future date the GSHP option is taken up, a further 81 bars may be put in (at $£ 478,800$ ) in the exposed grounds; the equipment will cost $£ 560,000$. Before GSHP, building energy will be supplied by conventional systems, same as with 'No Initial'.

\begin{tabular}{|c|c|c|}
\hline 1 All Initial & 2. No Initial & 3. Flex Initial \\
\hline Initial (and fixed) & $\Rightarrow$ After trigger & Initial $\Rightarrow$ After trigger \\
\hline $\begin{array}{l}\text { Initial: } 203 \text { bars }+ \text { HPs } \\
£ 1,760,000\end{array}$ & $\begin{array}{ll}\text { Initial: } 0 \text { bars } \Rightarrow & \text { After trigger: } \\
& +77 \text { bars }+ \text { HPs } \\
& \\
& £ 669,800\end{array}$ & $\begin{array}{ll}\text { Initial: } 122 \text { bars } \Rightarrow & \text { After trigger: } \\
& +81 \text { bars }+ \text { HPs } \\
& £ 1,038,800\end{array}$ \\
\hline
\end{tabular}

\section{Figure 1 - Principal test scenarios}

The model incorporates the uncertain exogenous conditions through Monte Carlo simulations, each over an expected building structure life-time of 50 years.

A dynamic system is used to evaluate annually the conditions for triggering the embedded options (scenarios 2 and 3) through the following four steps:

1 - Define probability distribution (PD) for each exogenous variable, i.e. gas and electricity prices, heating and cooling loads and the GSHP system's coefficient of performance (COP). The probability distribution of gas and electricity prices comes from the National Grid (2011) $)^{4}$ projections; the PD on heating and cooling loads are derived from estimates from GIEnergy ${ }^{5}$ and ASHRAE; ; the COP was given according to London's soil characteristics in London (mean value: 2.95), based on current research at Department of Engineering, Cambridge University.

2 - Define the trigger conditions for investors under Scenarios 2 and 3. The basic trigger is the overall financial saving which is defined as the difference between the savings on energy expenditure through using the GSHP (relative to using conventional systems) and the capital cost of the GSHP infrastructure and equipment. Any renewable energy subsidies may be included or excluded. If the saving is positive, the decision is triggered. The savings can be defined in terms of myopic evaluation (ME) - i.e. current year savings greater than annualised capital cost -, or in net present value evaluation (PVE) - i.e. comparing the savings and capital cost over 30 years in net present value (NPV); our main tests are based on the latter, NPV approach. In addition, we have tested alternative decision/implementation time-lag profiles in exercising flexible options (see Step 4 below).

3 - Monte Carlo simulation, which is to incorporate the probability distributions of exogenous variables and test the triggers dynamically over a period of 50 years which is our assumption of the working life of the office complex.

4 National Grid (2011). UK Future Energy Scenarios: UK gas and electricity transmission, November 2011

5 Gl Energy. Source: http://www.gienergy.net/ . Last access: March, 2014

6 ASHRAE (American Society Heating, Refrigerating and Air-conditioning Engineering). Source: https://www.ashrae.org/ . Last access: March, 2014 
Each test is performed 1,000 times, which according to our tests (from 500-10,000 runs) can adequately reveal the uncertainty profiles. The tests output a range of indicators that help decision-makers map cost-effective solutions.

4 - Sensitivity analyses. By changing the above mentioned test variables one at the time, a number of sub-scenarios have been tested. This includes excluding the Renewable Heating Incentives $(\mathrm{RHI})$, varying the discount rate from $6 \%$ down to $3 \%$ and up to $12 \%$, varying the energy prices, lowering the COP to account for inappropriate uses over time, and alternative decision/implementation time-lag profiles in exercising flexible options that is typical in business decision making processes. The tests represent a comprehensive range for identifying the roles played by each of the exogenous variables, the current cost-effecitive envelope for GSHP solutions, and possible measures to expand this envelope.

\section{TEST RESULTS SO FAR}

The model tests are still on-going, but they have proven that the GSHP-office building example is an effective case in testing our new approach. Results so far indicate that the cost ranking of the three principal scenarios is very sensitive indeed to the level of discount rates, the fluctuation of technical performance of the GSHP within the realistic range for London, the level of the Renewable Heat Incentive, and the timeliness of the decision-making process for GSHP installation/operation. Flex Initial (Scenario 3) has the promise of becoming the most cost-effective design under a remarkably wide range of circumstances relative to what has hitherto been considered. The cost-effective envelope of the flexible approach can be significantly expanded if the decision making processes and construction technology further improves. However, there are circumstances where either All Initial (Scenario 1) or No Initial (Scenario 2) ranks the most cost effective.

Under the 'central' projection of the exogenous variables, the mean total cost for building energy supply under Flex Initial is £2,136,830 in NPV, against £2,192,870 under All Initial and 2,254,820 under No Initial (Figure 2). The significant saving under Flex Initial is achieved through allowing investment in renewable energy to proceed when it is needed and not before. A substantial savings can be made whilst enabling construction to proceed as per demand for the office building.

The value of the flexible solution emerging from the results is due to the fact that, according to the probability distributions for uncertain elements, investing in the GSHP system is often expected to be more cost effective with embedded options than an option-free solution, if (1) gas and electricity prices continue to rise , (2) the Renewable Heat Incentive $(\mathrm{RHI})$ is in place, and (3) the technical performance of the GSHP is good (its COP=2.95 on average). Under the same circumstances doing nothing (i.e. under No Initial) is likely to cost more money, as well as consuming more gas and electricity.
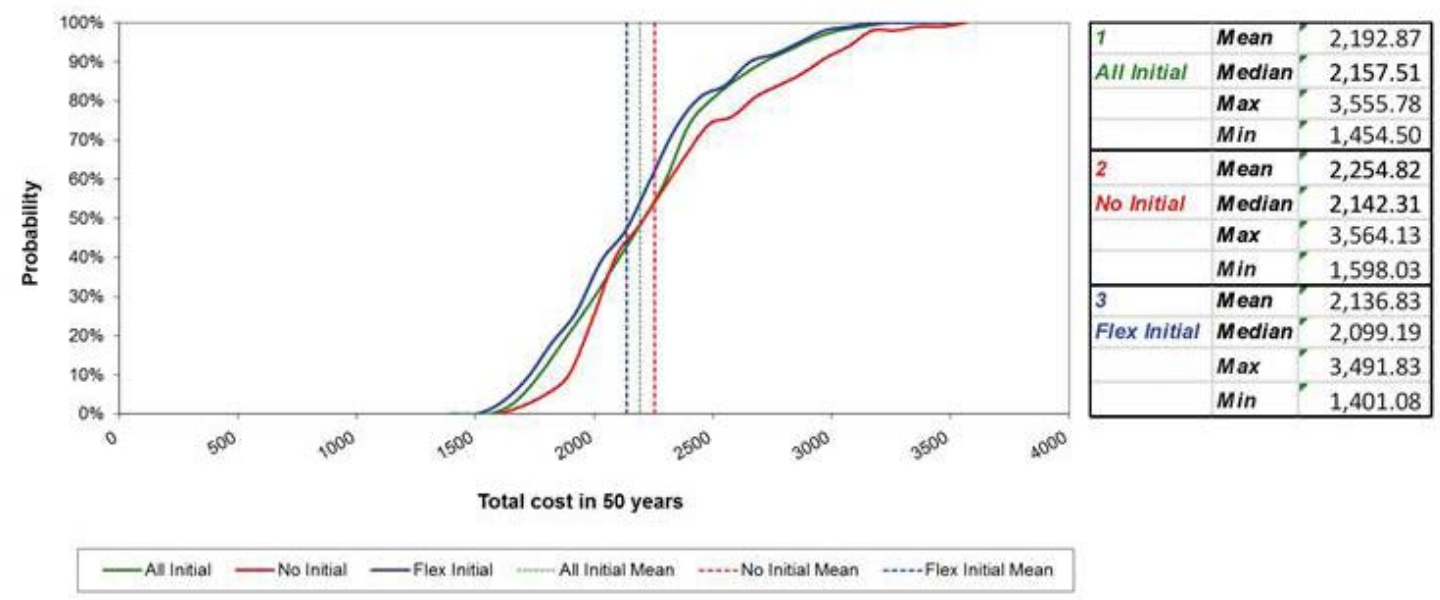

Figure 2 - Probability distribution of GSHP costs over a 50-year office building lifespan (1,000 Monte Carlo simulations) (NPV in $£ 1,000$ \& 2018 prices)

Simulations under all two trigger strategies show that results do not change substantially in using either myopic evaluation (ME) or present value evaluation (PVE) (Table 1).

Table 1 - Total net costs for building energy under myopic evaluation (ME) and present value evaluation (PVE) triggers (Results from 1,000 simulations, all values in Nin $£ 1,000$ \& 2018 prices) 


\begin{tabular}{|c|c|c|c|c|c|}
\hline & & & \multicolumn{3}{|c|}{ Design Scenarios } \\
\hline & & & All Initial & No Initial & Flex Initial \\
\hline \multirow{8}{*}{ 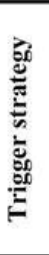 } & \multirow{4}{*}{ ME } & Mean & $2,200.39$ & $2,379.67$ & $2,143.39$ \\
\hline & & Median & $2,157.24$ & $2,258.00$ & $2,094.96$ \\
\hline & & Max & $3,634.15$ & $3,725.11$ & $3,577.68$ \\
\hline & & Min & $1,509.22$ & $1,662.21$ & $1,455.75$ \\
\hline & \multirow{4}{*}{ PVE } & Mean & $2,192.87$ & $2,254.82$ & $2,136.83$ \\
\hline & & Median & $2,157.51$ & $2,142.31$ & $2,099.19$ \\
\hline & & Max & $3,555.78$ & $3,564.13$ & $3,491.83$ \\
\hline & & Min & $1,454.50$ & $1,598.03$ & $1,401.08$ \\
\hline
\end{tabular}

Five sets of sensitivity analyses (as listed in Step 4) have been conducted. We summarise here the results under the PVE trigger, although in most cases the ME trigger gives very similar results. First, if the discount rate for net present value is lowered from $6 \%$ to $3 \%$, the cost ranking of the scenarios does not change but the difference between All Initial and Flex Initial will narrow. However, if the discount rate is raised to 12\%, No Initial becomes the lowest cost solution. Secondly, if the mean coefficient of performance (COP) of the GSHP system is lowered from 2.95 to 2.22 , which is at the low end for systems in the London region, the GSHP system will no longer be cost effective- in such cases No Initial ranks the cheapest. Thirdly, the current level of RHls appears to have been set at the right level to make the use of GSHPs financially competitive - removing the RHls will largely undermine the financial case for the use of GSHPs. Finally, even under favourable conditions to the GSHP, if the senior business managers delay exercising of the GSHP option when the financial triggers have been reached, the Flex Initial scenario can also become a less competitive scenario. In such conditions All Initial will emerge as a relatively cheap solution.

\section{CONCLUSIONS SO FAR}

The study shows that it is feasible to establish a future-option based model for testing in a realistic and robust way integrative designs of different types of infrastructure in terms of medium to long term costs and benefits such as typical in building and infrastructure construction. In particular, in our main case study, we have examined the integration of a GSHP system with an archetypal office building in dense urban areas, which is currently perceived to be a risky and unappealing investment in financial terms. Whether a significant net benefit can be achieved depends critically on a number of future external conditions that are difficult to predict in the decision-making process without a proper analytical tool. Our model has started to provide such a tool in managing those risks by taking account of the significant uncertainties and assessing different designs including those incorporating embedded future options. This accounts for both the benefits and costs of flexibility.

The new method presented in this paper has shown to be an effective way to assess the integrated infrastructure design, out of a set of possibilities. This will help minimise the risk of inefficient investment through adoption of flexible strategies. Indeed the results indicate that the flexible approach can be the most cost effective - particularly if there is proper governance in the decision-making process - because it can take advantage both from the use of a well performing GSHP system and from the financial savings due to the option of triggering the investment at the right time and not before.

The model is designed in particular to address the challenges arising from construction process conflicts, long expected life cycles and significant uncertainties in costs and decision-making processes. In future work we will extend the model for applications in the planning and designing of integrated complexes of buildings, transport facilities, renewable energy supply, water supply and waste management, such as found in dense urban areas. Indeed, similar to incorporating a GSHP system into a new building, other kind of infrastructures integration - such as configurations of building and transport facilities - also largely depends on the future configuration of the pertinent exogenous conditions (i.e: population, availability of public transport investment, fuel prices, etc). Typically, at the outset of planning and designing infrastructure facilities, comparatively little is known on the future configuration of the exogenous conditions such as operating costs and levels of use over their long design. The modeling approach proposed here contributes to the foundation of a more appropriate approach to planning, designing and assessing investment strategies in such circumstances. 